\title{
An Engineered Innate Repair Receptor Agonist, ARA 290, Protects Rat Islets from Cytokine-induced Apoptosis
}

Masaaki Watanabe', Yu Saito ${ }^{1}$, Jesper Wallmo', Tohru Takahashi', Randa A Hadi Diab², Ming Han Yao', Michael Brines ${ }^{3}$, Anthony Cerami ${ }^{1}$, ClaesGoran Ostenson ${ }^{4}$, Torbjorn Lundgren ${ }^{1}$ and Makiko Kumagai-Braesch ${ }^{1 *}$

${ }^{1}$ Division of Transplantation Surgery, CLINTEC, Karolinska Institutet Stockholm, Sweden

${ }^{2}$ Division of Clinical Immunology, Department of Laboratory Medicine, Karolinska Institutet, Stockholm, Sweden

${ }^{3}$ Araim Pharmaceuticals, Tarrytown, NY, USA

${ }^{4}$ Department of Molecular Medicine and Surgery, Karolinska Institutet, Stockholm, Sweden

\begin{abstract}
The efficacy of pancreatic islet transplantation (PITx) is reduced due to severe inflammatory responses triggered by the islet isolation and transplantation procedures. ARA 290, an innate repair receptor agonist, has anti-apoptotic and anti-inflammatory properties and it has been shown to improve clinical symptoms caused by inflammation in sarcoidosis patients and type 2 diabetes. We here investigated whether ARA 290 treatment would improve the efficacy of PITx in a rat pancreatic islet transplantation model. For islets co-cultured with proinflammatory cytokines, addition of ARA290 showed better viability [percent of naïve control in MTT assay: $54.6 \pm 5.2 \mathrm{vs.75.2} \pm 6.4$ ], insulin release [stimulation index in static glucose stimulated insulin secretion tests of $1.05 \pm 0.63$ vs. $2.61 \pm 0.89$ ], and reduced apoptosis [caspase $3 / 7$ activity $182 \pm 18$ vs. $152 \pm 18$ luminescence/dsDNA(ng)] (islets with cytokines vs. islets with ARA290+cytokines, respectively, all $p<0.05$ ). In order to mimic the clinical situation, islets were isolated after prolonged cold ischemia $(18 \mathrm{~h})$. The addition of ARA 290 into preservation solution, however, did not improve islet yields or function. As a marginal syngeneic PITx, 220 syngeneic rat islets were transplanted under the kidney capsule of streptozotocin-induced diabetic rats and the recipients were treated with ARA $290(120 \mu \mathrm{g} / \mathrm{kg} / \mathrm{day})$ for two weeks after PITx. In this model, no beneficial effect of ARA 290 treatment was observed. Our results indicated that ARA 290 protected rat pancreatic islets from cytokine-induced damage and apoptosis, but did not improve PITx perhaps due to poor penetration of ARA290 into transplant site or low IRR expression. ARA 290 could be useful as an agent to reduce islet injury caused by severe inflammation.
\end{abstract}

Keywords: Pancreatic islet transplantation; Innate repair receptor; Inflammation; Anti-apoptosis; Rat pancreatic islet isolation; Cold ischemia

Abbreviations: AUC: Area Under the Curve; dsDNA: Double
Stranded DNA; EPO: Erythropoietin; EPOR: Erythropoietin Receptor;
GSIS: Glucose-Stimulated Insulin Secretion; IRR: Innate Repair
Receptor; MTT:3-[4,5-Dimethylthiazol-2-yl]-2;5-Diphenyltetrazolium
Bromide; OGTT: Oral Glucose Tolerance Test; PBS: Phosphate Buffered
Saline; PITx: Pancreatic Islet Transplantation; POD: Postoperative Day
STZ: Streptozotocin

\section{Introduction}

Pancreatic islet transplantation (PITx) is a minimally invasive and potential curative procedure that can restore normoglycemia in type 1 diabetes patients without a major surgery or the complications associated with vascularized pancreas transplantation [1]. However, most of the islet allografts transplanted into the liver via the portal vein are subsequently destroyed following PITx [2-4]. Therefore, the PITx protocol requires transplant recipients to receive a large islet mass and often repeat transplantation in order to achieve insulin independence. In addition, the islet isolation process also triggers a cascade of stressful events in the islets involving apoptosis and the production of proinflammatory molecules that negatively influence islet function, producing detrimental effects following PITx [5-7]. Hence, a new strategy for preventing islet damage during isolation and at PITx is of great importance to improve transplant outcome and to enable successful transplantation even when using a lower islet mass.

To obtain acceptable islets for clinical use, the islets have to be separated from the acinar tissue of the harvested pancreas under clinical good manufacturing practice conditions. Despite the carefully controlled procedure, islets are exposed to mechanical, enzymatic, osmotic, and ischemic stresses [8-10], and the islet isolation process itself induces pro-inflammatory cytokines which are well known to cause islet graft damage [11,12]. A strong inflammatory response induced by islet isolation manifests as upregulation of tissue factor, monocyte chemoattractant protein (MCP)-1 [13] and inflammation associated genes such as interleukin-8 (IL-8), chemokine ligand 6, and compliment factor B [7]. In addition, the brain-death status of a donor, which induces a cytokine storm, organ procurement procedures, and prolonged cold ischemia time reduces isolated pancreatic islet yields and functionality after PITx [14-16]. Beyond the direct toxic effect on $\beta$-cells [17], inflammatory mediators, such as tumor necrosis factor (TNF)- $\alpha$ [18] IL-1 $\beta$ [19] and MCP-1 [20], may damage the transplanted islets by enhancing inflammation and innate immune responses following PITx. These mechanisms play a crucial role in the triggering of graft dysfunction and the eventual loss of islets after PITx.

Notably, inflammation induces the expression in diverse cells of

*Corresponding author: Masaaki Watanabe/Makiko Kumagai-Braesch, Division of Transplantation Surgery, CLINTEC, Karolinska Institutet Stockholm Sweden, Tel: +46733646121; Fax: +4687743 91; E-mail: masaaki@w8.dion.ne.jp, makiko.kumagai-braesch@ki.se

Received September 13, 2016; Accepted October 15, 2016; Published October 23, 2016

Citation: Watanabe M, Saito Y, Wallmo J, Takahashi T, Diab RAH, et al. (2016) An Engineered Innate Repair Receptor Agonist, ARA 290, Protects Rat Islets from Cytokineinduced Apoptosis. J Diabetes Metab 7: 708. doi: 10.4172/2155-6156.1000708

Copyright: (C) 2016 Watanabe M, et al. This is an open-access article distributed under the terms of the Creative Commons Attribution License, which permits unrestricted use, distribution, and reproduction in any medium, provided the original author and source are credited. 
a cytokine receptor complex which exerts anti-inflammatory, cytoprotective, and reparative effects [21]. This receptor is a heterodimeric receptor consisting of erythropoietin receptor (EPOR) subunit and $\mathrm{CD} 131$ the $\beta$-common subunit (EPOR- $\beta \mathrm{cR}$ ) [22,23]. This heteroreceptor been called as the Innate Repair Receptor (IRR) and is expressed by a variety of cells, including pancreatic islets [24,25], following cellular stress. ARA 290, also called pyroglutamate helix B surface peptide, is a newly developed specific IRR agonist. ARA 290 has been tested clinically and showed improvement of clinical symptoms caused by inflammation, such as neuropathic pain in sarcoidosis and improved metabolic control of type 2 diabetes [26,27].

The rat pancreatic islets express IRR when exposed proinflammatory cytokines and treatment with human recombinant EPO reduced apoptosis and maintained the function [24]. Muller et al. also demonstrated that ARA 290 improves islet function of GK rat, model for type 2 diabetes [28]. We have demonstrated using a mouse system that ARA 290 protects isolated mouse pancreatic islets from cytokine induced damage and apoptosis, inhibits mouse macrophage activity, and improves the engraftment of mouse syngeneic islets transplanted into the liver via the portal vein [29].

In the present study, we investigated the effects of ARA 290 on rat islet isolation procedure and on isolated islets, and then evaluated the efficacy of ARA 290 in a rat syngeneic PITx model transplanted under the kidney capsule.

\section{Materials and Methods}

\section{Animals}

Lewis rats (Charles River, Sulzfeld, Germany) were maintained in a specific pathogen-free facility at Karolinska Institutet, Stockholm, Sweden. All experiments were approved by the local ethics committee, and the study was conducted according to the Guidelines for the Care and Use of Laboratory Animals of Karolinska Institutet.

\section{Reagents}

An engineered Innate Repair Receptor agonist, ARA 290, was provided by Araim Pharmaceuticals, Inc. (Tarrytown, NY). ARA 290 stock solution ( 1 or $2 \mathrm{mg} / \mathrm{mL}$ ) was dissolved in phosphate buffered saline (PBS), filter sterilized $(0.2 \mu \mathrm{m})$ and maintained at $4^{\circ} \mathrm{C}$ for up to 4 weeks. Recombinant rat interleukin (IL)- $1 \beta$, interferon (IFN)- $\gamma$ and tumour necrosis factor (TNF)- $\alpha$ were purchased from PeproTech Nordic (Stockholm, Sweden).

\section{Pancreatic islet isolation and culture}

The pancreatic islets of Lewis rats (300-350 g) were isolated as previously described [30]. Briefly, the rat pancreases were digested using collagenase P solution $(0.7 \mathrm{mg} / \mathrm{mL}$; Roche Diagnostics $\mathrm{GmbH}$, Mannheim, Germany) and the islets were purified using discontinuous density gradient centrifugation (Histopaque-1119, Histopaque-1077, Sigma-Aldrich). In one study, pancreatic islets were also isolated from the harvested pancreas after preserved under cold ischemia for $18 \mathrm{hrs}$ as previously described by Pileggi et al. [31]. Briefly, rats were anesthetized by inhalation of isoflurane (Baxter medical AB, Kista, Sweden). The abdominal organs were perfused through the aorta using cold University of Wisconsin (UW) solution (Apoteket, Sweden), and the pancreas was removed en-bloc with the spleen and duodenum and put into the cold UW solution. After $18 \mathrm{hrs}$ of cold preservation $\left(4^{\circ} \mathrm{C}\right)$, the harvested pancreas was distended with collagenase $\mathrm{P}$ solution and digested in the same method described above.
Isolated islets were cultured in RPMI 1640 medium (Gibco, BRL, Life Technology Ltd., Scotland) supplemented with $10 \%$ fetal bovine serum (FBS), $2 \mathrm{mmol} / \mathrm{L}$ L-glutamine, $100 \mathrm{U} / \mathrm{mL}$ penicillin and 100 $\mu \mathrm{g} / \mathrm{mL}$ streptomycin (all provided by Life Technologies Europe BV, Stockholm, Sweden) at $37^{\circ} \mathrm{C}$ with $5 \% \mathrm{CO}_{2}$ in a humidified atmosphere. Islets were cultured for $20 \mathrm{hrs}$ prior to islet transplantation and $36 \mathrm{hrs}$ prior to the in vitro study.

\section{Pancreatic islet incubation with cytokines}

The isolated islets were transferred to a $35-\mathrm{mm}$ cell culture dish (Medicarrier AB, Spånga, Sweden) containing culture medium described above. In order to mimic the inflammatory condition during clinical transplantation, the islets were exposed to rat proinflammatory cytokines: IL- $1 \beta(0,5 \mathrm{ng} / \mathrm{mL}$, corresponding to $50 \mathrm{units} / \mathrm{mL})$, IFN- $\gamma$ (100 ng/ML corresponding to 1,000 units/mL) and TNF- $\alpha(50 \mathrm{ng} / \mathrm{mL}$ corresponding to 1,000 units $/ \mathrm{mL}$ ) for $6 \mathrm{hrs}$ at $37^{\circ} \mathrm{C}$ with $5 \% \mathrm{CO}_{2}$ in a humidified atmosphere. During the culture, ARA $290(100 \mathrm{nmol} / \mathrm{L})$ was added, and cell viability, apoptosis and functions were assessed.

\section{Measurement of caspase $3 / 7$ activity}

Apoptosis was assayed by using the Caspase-Glo 3/7 Assay kit (Promega Corp., Madison, WI). In total, 100 islets were suspended in $100 \mu \mathrm{L}$ of RPMI-1640 medium and sonicated for 10 seconds. According to the manufacturer's instructions, $100 \mu \mathrm{L}$ of CaspaseGlo 3/7 reagent was added and incubated at room temperature for one hour. The luciferase activity was measured with a luminometer (Biotek FLx $800^{\text {mix }}$ Multi-Detection Microplate Reader operated by Gen $5^{\text {Tx }}$ Data Analysis Software). In order to correct the amount of cells in each sample, the amount of double stranded DNA (dsDNA) was measured by dyeing the sample with the Quant-iT ${ }^{\mathrm{m} m}$ PicoGreen dsDNA Assay Kit (Invitrogen, Carlsbad, CA) according to the manufacturer's instructions. Fluorescence activity was measured with a fluorometer (Biotek FLx800 ${ }^{\text {mi }}$ Multi-Detection Microplate Reader operated by Gen5 ${ }^{\text {Tix }}$ Data Analysis Software).

\section{Viability tests by MTT assay}

The islet cell viability was assessed by MTT assay. Twenty islets were collected and suspended in $200 \mu \mathrm{L}$ of culture medium and dispensed into round-bottomed 96-well plates. The 3-(4,5-dimethylthiazol-2-yl)-2,5diphenyltetrazolium bromide tetrazolium (MTT; Sigma-Aldrich) was dissolved in PBS $(5 \mathrm{mg} / \mathrm{mL})$ and the MTT assay performed as described previously [32]. Absorbance of the converted dye was measured at a wavelength of $550 \mathrm{~nm}$ on a Biotek FLx800 ${ }^{\mathrm{mm}}$ Multi-Detection Microplate Reader operated by Gen $5^{\text {ma }}$ Data Analysis Software

\section{Static glucose-stimulated insulin secretion tests}

Twenty islets were cultured in a $35-\mathrm{mm}$ dish containing $3 \mathrm{~mL}$ of RPMI-1640 medium with or without pro-inflammatory cytokines, and with or without ARA $290(100 \mathrm{nmol} / \mathrm{L})$ for $6 \mathrm{hrs}$ as described above. All the islets were hand-picked and transferred to 24-well Transwell plates $(8.0 \mu \mathrm{m}$ pore size membrane, Corning, Acton, MA) in $1.5 \mathrm{~mL}$ of Krebs-Ringer bicarbonate buffer (KRBB) containing $1.67 \mathrm{mmol} / \mathrm{L}$ glucose (low glucose KRBB). The islets were incubated for $60 \mathrm{~min}$ with $1.5 \mathrm{~mL}$ of low glucose $\mathrm{KRBB}$, followed by incubation with high glucose KRBB (glucose $16.7 \mathrm{mmol} / \mathrm{L}$ ) for another $60 \mathrm{~min}$. The supernatants were collected and the insulin in the supernatants was quantitated using a rat insulin enzyme-linked immunosorbent assay (ELISA) kit (Mercodia, Uppsala, Sweden). A stimulation index was calculated by dividing the total amount of insulin released from the islets cultured in the high-glucose KRBB by the total amount of insulin released from the islets cultured in the low-glucose KRBB. 


\section{Diabetes mellitus induction}

Female Lewis rats were rendered diabetic by a single intravenous injection of streptozotocin $(55 \mathrm{mg} / \mathrm{kg})$ four days prior to PITx. Blood samples were collected via the tail vein, and the blood glucose levels were monitored with the Accu-Check blood glucose monitor (Medicarrier). Diabetes was considered to be established when the blood glucose level of two consecutive measurements exceeded $20 \mathrm{mmol} / \mathrm{L}(360 \mathrm{mg} / \mathrm{dL})$.

\section{Syngeneic marginal rat PITx}

To determine the optimal number of islets for marginal PITx, the isolated rat syngeneic pancreatic islets of $600,500,400,300,250$ or 150 were transplanted under the kidney capsule of diabetic recipient rats as a preliminary PITx. The normoglycemic rates after PITx were $100 \%$ in the recipient animals transplanted with more than 250 islets, and any recipient animals did not became normoglycemic with 150 islets (data not shown). The marginal islet number was determined to be 220 islets based on the preliminary PITx. The isolated 220 rat pancreatic islets were washed once with HBSS and packed into a 24 GA VenflonTM (BD) using a Hamilton syringe, and were transplanted under the left kidney capsule of diabetic recipient rats.

\section{ARA 290 treatment protocol}

On the day of PITx, ARA $290(60 \mu \mathrm{g} / \mathrm{kg})$ was administered intraperitonealy at 0 and $2 \mathrm{hrs}$ before PITx, and subcutaneously (120 $\mu \mathrm{g} / \mathrm{kg})$ at 6 and $12 \mathrm{hrs}$ after PITx. The same dose of ARA $290(120 \mu \mathrm{g} /$ $\mathrm{kg}$ ) was administered subcutaneously twice a day from postoperative day (POD) 1 to 7 , and once a day from POD 8 to 14 . The same volume of PBS was administered to control group animals.

\section{Post-transplant management}

Non-fasting blood samples were collected via the tail vein, and the blood glucose levels were monitored prior to and then daily for up to 30 days after PITx. Normoglycemia was defined when the blood glucose level was reduced to below $11.1 \mathrm{mmol} / \mathrm{L}(200 \mathrm{mg} / \mathrm{dL})$ on two consecutive days. The islet graft bearing kidney was collected, and homogenised in acid-ethanol for insulin extraction. Insulin content was measured by rat insulin ELISA kit (Mercodia, Uppsala, Sweden).

\section{Oral glucose tolerance test (OGTT)}

An OGTT was performed at four weeks after PITx. Glucose (1.5 g/ $\mathrm{kg}$ ) was administered orally to the rats after $16 \mathrm{hrs}$ of fasting, and the blood glucose levels were monitored immediately before and at $0,5,10$, $15,20,30,45,60,90$, and 120 minutes after the glucose administration. The insulin levels in the plasma were also measured at $0,10,20,30,45$, and 60 minutes after the glucose administration by using a rat insulin ELISA kit (Mercodia).

\section{Insulin extraction from graft-bearing kidneys}

Graft bearing kidneys were harvested 30 days after transplantation. The transplanted islet grafts were identified macroscopically and the entire region of each transplanted graft on the kidney was taken out and fixed with $1 \mathrm{~mL}$ of acid ethanol solution. The tissue was kept at $-20^{\circ} \mathrm{C}$ for over night and homogenized. Another $20 \mathrm{hrs}$ incubation at $-20^{\circ} \mathrm{C}$, unbroken tissues were spun down and the supernatant collected. Prior to ELISA assay, the solution was neutralized with the same volume of Tris Buffer ( $\mathrm{pH} 7.5)$, Insulin content was measured by Rat insulin ELISA kit from Mercodia.

\section{Statistical analysis}

Quantitative results are presented as mean values \pm standard deviation (SD). Statistical analysis between two groups was carried out using the Mann-Whitney $U$-test. When there are three groups, Kruskal-Wallis tests with Dunn's multiple comparison test were used. Differences in the normoglycemic rate between groups were evaluated by log-rank test using Kaplan-Meier survival curves. A $p$-value< 0.05 was considered statistically significant, and all calculations were performed using GraphPad Prism software version 6 (GraphPad Software Inc., San Diego, CA).

\section{Results}

\section{ARA 290 protects isolated rat pancreatic islets from cytokine-} induced damage and apoptosis

The pancreatic islet isolation procedure exerts significant stress on islets by releasing pro-inflammatory cytokines that result in loss of $\beta$-cell function and induction of apoptosis [17-19]. We initially investigated the tissue protective effects of ARA 290 in an in vitro rat islet-culture model. Isolated rat pancreatic islets were cultured together with pro-inflammatory cytokine cocktail (IL-1 $\beta$, TNF- $\alpha$, and IFN- $\gamma$ ) in the presence or absence of ARA 290 for $6 \mathrm{hrs}$ at $37^{\circ} \mathrm{C}$. The viability and function of islets were assessed by MTT assay and static glucose-stimulated insulin secretion tests. The addition of ARA 290 improved islet viability during the exposure to pro-inflammatory cytokines. In the MTT assay, the absorbance of islets cultured with proinflammatory cytokine or ARA 290 plus cytokines was compared to those of islets cultured with medium alone (54.6 \pm 5.2 vs. $75.2 \pm 6.4 \%$ of naïve control, $\mathrm{p}<0.05$, respectively, $\mathrm{n}=4$, Figure $1 \mathrm{~A}$ ) The cytokineinduced suppression of glucose-stimulated insulin secretion was partially counteracted by ARA 290 (Figure 1B). To evaluate the antiapoptotic effects of ARA 290, the activity of caspase $3 / 7$ in the cultured islets was examined. The caspase 3/7 activity increased in the islets cocultured with the proinflammatory cytokines. In contrast, the addition of ARA 290 significantly suppressed caspase $3 / 7$ activity indicating that ARA 290 protects pancreatic islets from cytokine-induced apoptosis. The beneficial effect was clearly seen at 6 hrs after exposure of cytokines (Figure 1C).

Addition of ARA 290 during the cold preservation period or in the collagenase solution did not affect islet yields, viability, or function

Prolonged cold ischemia has been well known to reduce islets viability, function, and also islet yield in animal models [33] and clinical setting [15]. We then evaluated whether ARA 290 protects islets from cold ischemia related islet damage. ARA 290 was added into the UW solution during cold preservation, and its effects in islet isolation were evaluated. As shown in Figures 2A-2D, there was no clear difference between the ARA 290 and control groups in islet yields, viability, or function. The effect on ARA 290 on islet isolation without prolonged cold ischemia was also evaluated by using rat pancreas in which collagenase solution with or without ARA 290 was infused into the harvested pancreas without prolonged cold ischemia. There was no significant difference between the groups with or without ARA 290 (data not shown)

\section{No beneficial effect of ARA 290 administration on rat islet graft function transplanted under kidney capsule}

In order to evaluate the effect of ARA 290 treatment on PITx, we used a rat syngeneic marginal PITx model. The marginal mass of rat islets (220 islets) was transplanted under the kidney capsule of STZ induced diabetic rats, and the recipient animals were treated with ARA 
A

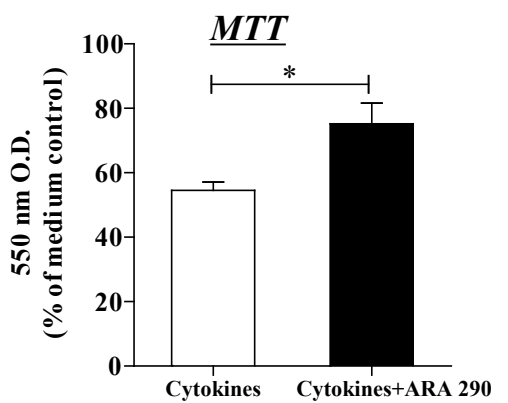

$\mathrm{B}$

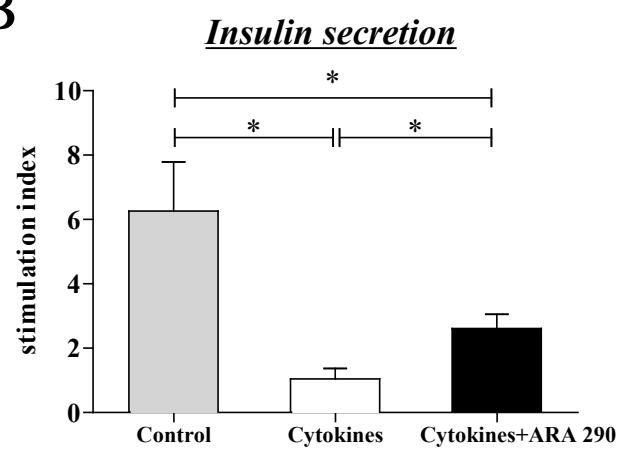

C

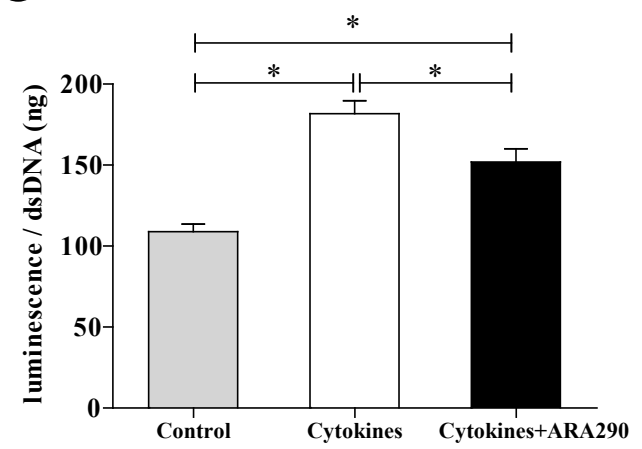

Figure 1: ARA 290 protects isolated rat pancreatic islets from cytokineinduced damage and apoptosis. The isolated pancreatic islets were cocultured together with mixture of IL-1 $\beta$, TNF- $\alpha$, and IFN-y in the presence or absence of ARA 290 for 6 hours. (A) In an MTT assay, relative optical density (O.D.) values compared to those of islets cultured with medium alone are shown (mean \pm SD). The addition of ARA 290 maintained islet viability during culturing together with pro-inflammatory cytokines ( $p<0.05, n=4$, MannWhitney U-test, respectively). (B) The insulin secretion from the co-cultured islets was partially recovered when ARA 290 was present. The stimulation index (SI.) was calculated as a ratio of the insulin value after high glucose stimulation divided by that of low glucose stimulation (mean \pm SD) ( $(p<0.05$, $n=4$ ). (C) The addition of ARA 290 significantly suppressed the caspase $3 / 7$ activity at $6 \mathrm{~h}$ after co-culturing with the cytokines compared to the vehicletreated control group ( $p<0.05, n=5)$. ' $n$ ' indicates different batches of islets.

290 for two weeks following PITx. Non-fasting blood glucose levels are shown in Figures 3A and 3B (control group and ARA 290 treated group, respectively). No significant differences were observed between the two groups in comparison of normoglycemic rate, non-fasting blood glucose levels, body weight gain during 30 days, and insulin content of the grafts (Figure 3C).

To further characterize the function of transplanted islet grafts, oral
A

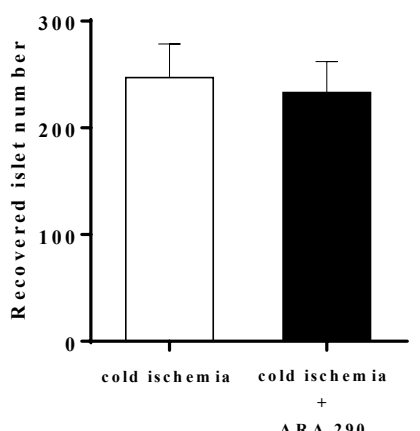

B

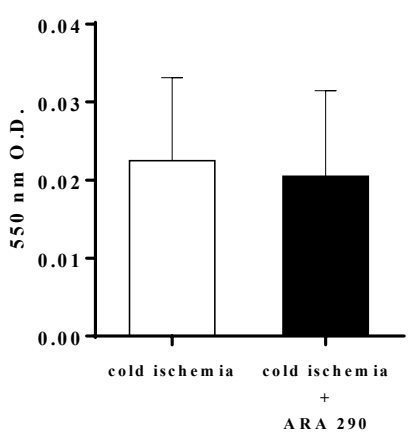

C

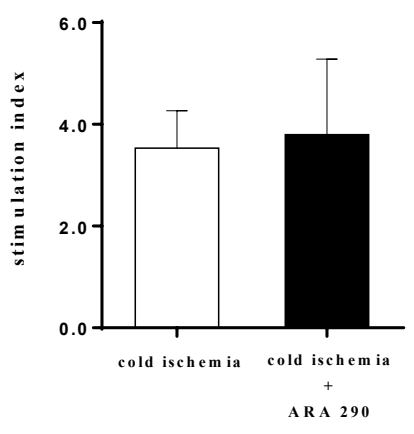

D

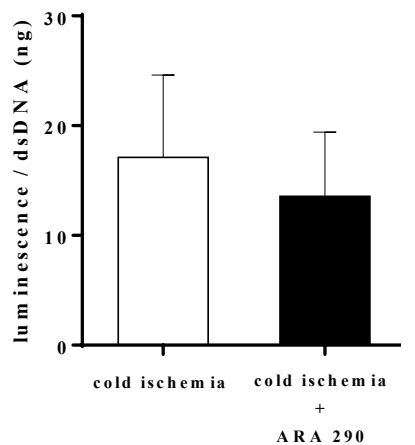

Figure 2: The effect of ARA 290 treatment on islet isolation with prolonged cold ischemia. ARA $290(100 \mathrm{nM})$ was added in cold preservation solution (UW solution) and also collagenase solution, and the results are compared without ARA 290 group. The yields of isolated pancreatic islets in each group are shown in A. The MTT assay was performed as a viability test. The O.D. values are shown in B. Islets function was evaluated in the insulin secretion in response to high glucose. Stimulation Index, calculated as insulin amount in high glucose divided by insulin amount in low glucose, is shown in C. Caspase $3 / 7$ activity (luminescence)/dsDNA (ng) was assessed as indication of apoptotic cells in D. Each value is represented as mean \pm SD. No significant differences were observed between each group in islet yields, caspase $3 / 7$ activity, viability or function ( $n=5$ in all experiments). 
A

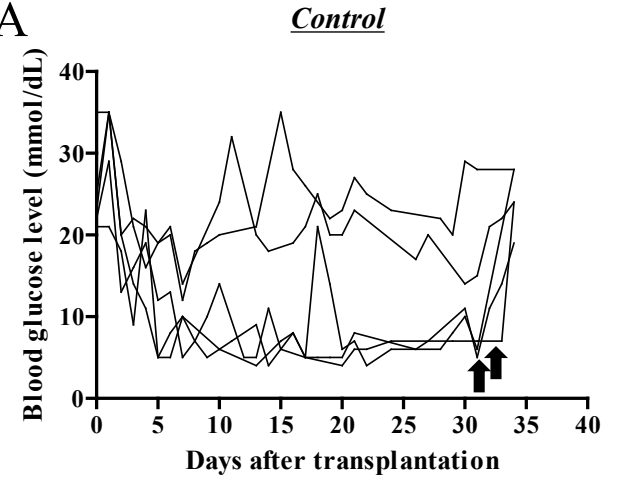

$\mathrm{B}$

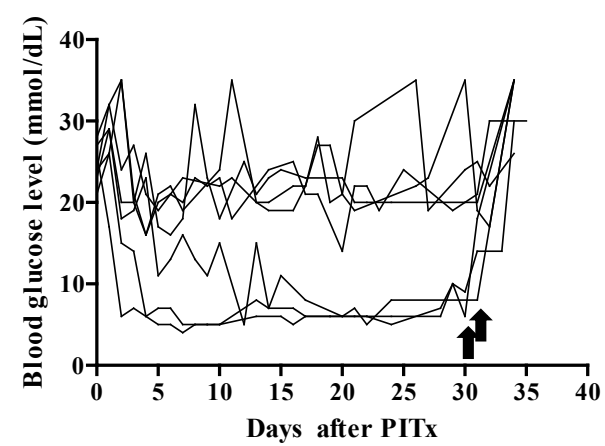

C

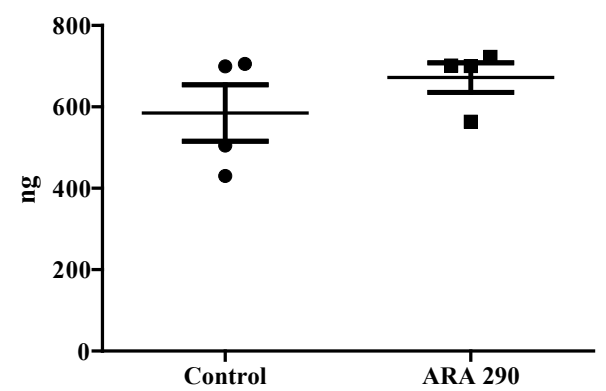

Figure 3: Islet graft functions following syngeneic marginal PITx. Two hundred and twenty syngeneic rat islets were transplanted under kidney capsules, and non-fasting blood glucose level was measured. A graft-bearing nephrectomy was performed approximately 30 days after transplantation (arrows). Blood glucose levels in control group $(A, n=5)$ and ARA 290 treated group are shown $(B, n=7)$. No significant differences were observed between the two groups in normoglycemic rate (log-rank test). A subset of the graftbearing kidneys was harvested and insulin was extracted. Insulin content/ kidney is shown in each group. There was no difference between ARA 290 treated and non-treated groups $(C: n=4)$.

glucose tolerance test (OGTT) was performed at four weeks after PITx. Blood glucose curves were similar between the two groups (Figure 4A), and area under the curve (AUC) of blood glucose and plasma insulin level during the OGTT showed no clear differences between the groups (Figures 4B and 4C).

\section{Discussion}

The process of pancreatic islet isolation and the severe inflammatory reaction associated with the PITx procedure leads to release of pro-inflammatory cytokines and free radicals, which directly damage transplanted islets and induce apoptosis of [34]. In the current study, rat islets were exposed to high doses of pro-inflammatory cytokines in vitro to mimic the inflammatory reactions caused by the PITx procedure. We found that ARA 290 maintained the viability and function of isolated rat pancreatic islets during culture in the presence of pro-inflammatory cytokines. The addition of ARA 290 significantly suppressed caspase 3/7 activity after co-culturing with proinflammatory cytokines compared to the vehicle-treated control group. Mechanistically, ARA 290 exerts anti-inflammatory, antiapoptotic, and tissue-protective effects through its interaction with the IRR [35-37] and subsequently suppresses the nuclear factor- $\mathrm{kB}$ driven gene transcription of pro-inflammatory mediators, which leads to the phosphorylation of endothelial nitric oxide synthase and other signaling systems $[35,38,39]$. Exposure of proinflammatory cytokines causes IRR expression on the cell surface [24] and therefore the addition of ARA 290 could activate this receptor to prevent cytokine-induced damage and apoptosis.
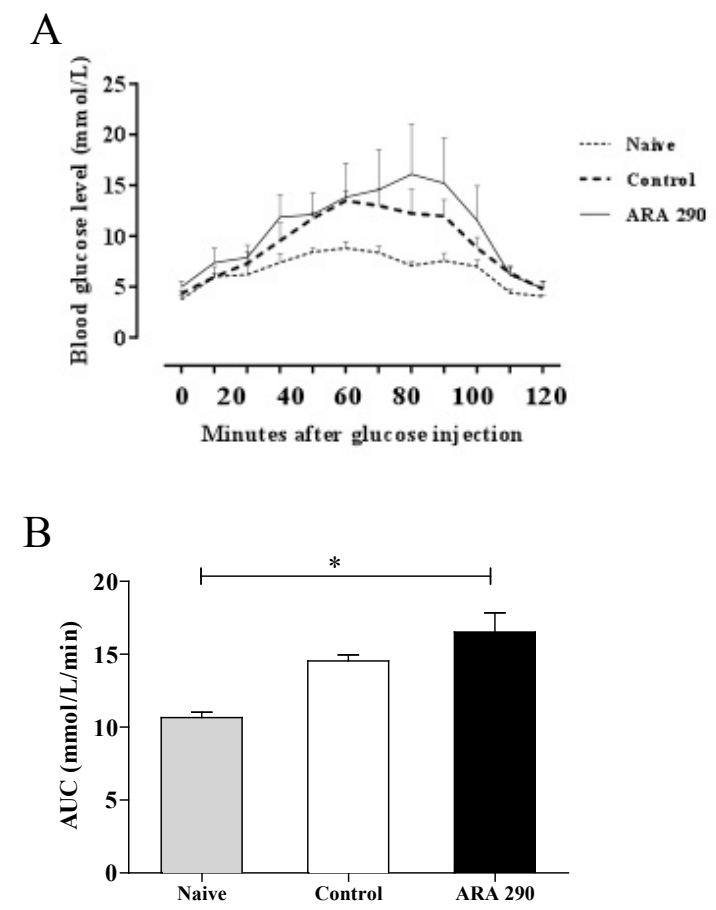

C

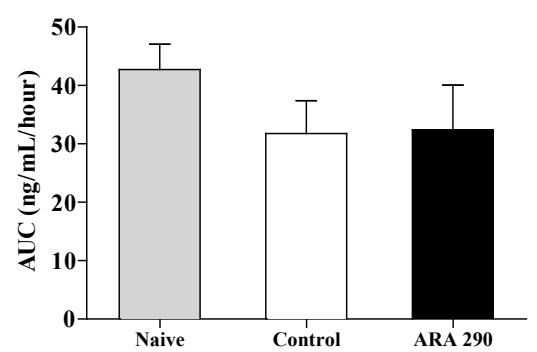

Figure 4: Islet graft functions of the syngeneic marginal mass of islets transplantation model. An OGTT was performed at four weeks after PITx (A and $B$ ). The blood glucose levels and the AUC during IPGTT of naïve control $(n=3)$, vehicle-treated control $(n=5)$, and ARA 290-treated recipients $(n=7)$ are shown. (C) Plasma insulin levels during OGTT in each group are shown (mean \pm SD). Statistical differences were observed in AUC-blood glucose between naïve and ARA 290 treated PITx rat ( $p<0,05)$. No significant differences were observed between the groups in the AUC of glucose or insulin. 
These results are in line with our findings using mouse pancreatic islets which showed that ARA 290 protected islets from cytokineinduced damage and apoptosis [29]. Additionally, ARA 290 treatment inhibited mouse macrophage activation in an in vitro experiment, which suggested that ARA 290 treatment could prevent inflammatory responses elicited by the PITx procedure via the portal vein route [29]. In the current study, the addition of ARA 290 during a long-term cold preservation and collagenase digestion did not show any improvement in islet yield, viability, and function. This may be explained by lack of IRR expression in the setting of low temperature and will require further study. Although prolonged cold ischemia is reported to be associated with lower islet yields and viability, as well as the function of transplanted islet [31], the pancreases used in our study were obtained from non-brain dead donors and therefore were not subjected to the cytokine storm arising from brain death [16].

Moreover, in the marginal mass of rat syngeneic PITx, there was no clear effect of ARA 290 treatment. Islets transplanted under the kidney capsule are characterized initially by having a very low oxygen tension, due to a lack of vascular supply, with perfusion being restored by neovascularization beginning only after several days and reaching completion at 1 month following transplantation [40]. ARA 290, as a small peptide, has a very short plasma half-life of several minutes. It is quite likely that the rapid clearance of ARA 290 coupled with the lack of intact microcirculation at the transplant site prevents adequate diffusion of this IRR agonist to the islets. Additionally, in the current study, syngeneic rat islets were transplanted under the kidney capsule of recipient animals, a procedure which does not elicit an IBMIR (instant blood mediated inflammatory reaction) [41]. The inflammatory responses after PITx via this route are relatively mild and might not be sufficient to increase IRR expression $[42,43]$. The discrepancy between the mouse study [29] and our current results may be explained by differences in the degree of inflammation and/or islet damage which is associated with the expression of the IRR in conjunction with the poor perfusion of the islets at the transplant site. Although we could not clearly establish the mechanism of action of ARA 290, continued investigation in the relevance between the expression of IRR and the efficacy of ARA 290 could provide the opportunity to expand and deepen our knowledge on islet damage following PITx. These studies are currently in progress.

\section{Conclusion}

This study confirmed that ARA 290 protects rat pancreatic islet subjected to severe inflammation. Synthesizing the results from both this study and a recently published mouse study [29], ARA 290 could be used as an agent for prevention of islet damage caused by severe inflammation.

\section{Acknowledgement}

We thank to Dr. Carole Muller, Karolinska Institutet, for fruitful discussion and skillful support in experiments.

\section{Funding}

This study was supported in part by the regional agreement on medical training and clinical research (ALF) between Stockholm County Council and the Karolinska Institutet, the Strategic Research Program in Diabetes at Karolinska Institutet (CLINTEC, H9), the Foundation for International Surgical Cooperation, The Swedish Child Diabetes Foundation, the Swedish Diabetes Association and the Swedish Research Council.

\section{Disclosure}

M.B. and A.C. are the stockholders of Araim Pharmaceuticals, Tarrytown, NY, US, which provided ARA 290. All other authors declare no conflicts of interest.

\section{References}

1. Ryan EA, Lakey JR, Paty BW, Imes S, Korbutt GS, et al. (2002) Successful islet transplantation: continued insulin reserve provides long-term glycemic control. Diabetes 51: 2148-2157.

2. Bennet W, Sundberg B, Groth CG, Brendel MD, Brandhorst D, et al. (1999) Incompatibility between human blood and isolated islets of Langerhans: a finding with implications for clinical intraportal islet transplantation? Diabetes 48: 1907-1914.

3. Eich T, Eriksson O, Sundin A, Estrada S, Brandhorst D, et al. (2007) Positron emission tomography: a real-time tool to quantify early islet engraftment in a preclinical large animal model. Transplant 84: 893-898.

4. Eich T, Eriksson O, Lundgren T, Nordic Network for Clinical Islet Transplantation (2007) Visualization of early engraftment in clinical islet transplantation by positron-emission tomography. N Engl J Med 356: 2754-2755.

5. Blinman TA, Gukovsky I, Mouria M, Zaninovic V, Livingston E, et al. (2000) Activation of pancreatic acinar cells on isolation from tissue: cytokine upregulation via p38 MAP kinase. Am J Physiol Cell Physiol 279: C1993-C2003.

6. Paraskevas S, Maysinger D, Wang R, Duguid TP, Rosenberg L (2000) Cell loss in isolated human islets occurs by apoptosis. Pancreas 20: 270-276.

7. Negi S, Jetha A, Aikin R, Hasilo C, Sladek R, et al. (2012) Analysis of beta-cell gene expression reveals inflammatory signaling and evidence of dedifferentiation following human islet isolation and culture. PloS ONE 7: e30415.

8. Cattan P, Berney T, Schena S, Molano RD, Pileggi A, et al. (2001) Early assessment of apoptosis in isolated islets of Langerhans. Transplantation 71 : $857-862$.

9. Thomas F, Wu J, Contreras JL, Smyth C, Bilbao G, et al. (2001) A tripartite anoikis-like mechanism causes early isolated islet apoptosis. Surgery 130: 333-338.

10. Wang RN, Rosenberg $L$ (1999) Maintenance of beta-cell function and survival following islet isolation requires re-establishment of the islet-matrix relationship. J Endocrinol 163: 181-190.

11. Bottino R, Balamurugan AN, Tse H, Thirunavukkarasu C, Ge X, et al. (2004) Response of human islets to isolation stress and the effect of antioxidant treatment. Diabetes 53: 2559-2568.

12. Johansson U, Olsson A, Gabrielsson S, Nilsson B, Korsgren O (2003) Inflammatory mediators expressed in human islets of Langerhans: implications for islet transplantation. Biochem Biophys Res Commun 308: 474-479.

13. Bertuzzi F, Marzorati S, Maffi P, Piemonti L, Melzi R, et al. (2004) Tissue factor and $\mathrm{CCL} 2 /$ monocyte chemoattractant protein-1 released by human islets affect islet engraftment in type 1 diabetic recipients. J Clin Endocrinol Metab 89 5724-5728.

14. Contreras JL, Eckstein C, Smyth CA, Sellers MT, Vilatoba M, et al. (2003) Brain death significantly reduces isolated pancreatic islet yields and functionality in vitro and in vivo after transplantation in rats. Diabetes 52: 2935-2942.

15. Hilling DE, Bouwman E, Terpstra OT, Marang-van de Mheen PJ (2014) Effects of donor-, pancreas-, and isolation-related variables on human islet isolation outcome: a systematic review. Cell Transplant 23: 921-928.

16. Saito Y, Goto M, Maya K, Ogawa N, Fujimori K, et al. (2010) Brain death in combination with warm ischemic stress during isolation procedures induces the expression of crucial inflammatory mediators in the isolated islets. Cell Transplant 19: 775-782.

17. Bottino R, Fernandez LA, Ricordi C, Lehmann R, Tsan MF, et al. (1998) Transplantation of allogeneic islets of Langerhans in the rat liver: effects of macrophage depletion on graft survival and microenvironment activation. Diabetes 47: 316-323.

18. Satoh M, Yasunami Y, Matsuoka N, Nakano M, Itoh T, et al. (2007) Successfu islet transplantation to two recipients from a single donor by targeting proinflammatory cytokines in mice. Transplant 83: 1085-1092.

19. Arnush M, Heitmeier MR, Scarim AL, Marino MH, Manning PT, et al. (1998) IL-1 produced and released endogenously within human islets inhibits beta cell function. J Clin Invest 102: 516-526.

20. Piemonti L, Leone BE, Nano R, Maffi P, Bianchi G, et al. (2002) Human pancreatic islets produce and secrete MCP-1/CCL2: relevance in human islet transplantation. Diabetes 51: 55-65. 
Citation: Watanabe M, Saito Y, Wallmo J, Takahashi T, Diab RAH, et al. (2016) An Engineered Innate Repair Receptor Agonist, ARA 290, Protects Rat Islets from Cytokine-induced Apoptosis. J Diabetes Metab 7: 708. doi: 10.4172/2155-6156.1000708

21. Youssoufian H, Longmore G, Neumann D, Yoshimura A, Lodish HF (1993) Structure, function, and activation of the erythropoietin receptor. Blood 81 : 2223-2236.

22. Brines M, Cerami A (2006) Discovering erythropoietin's extra-hematopoietic functions: biology and clinical promise. Kidney Int 70: 246-250.

23. Zhang YL, Radhakrishnan ML, Lu X, Gross AW, Tidor B, et al. (2009) Symmetric signaling by an asymmetric 1 erythropoietin: 2 erythropoietin receptor complex. Mol Cell 33: 266-274

24. Fenjues ES, Ochoa MS, Cabrera O, Mendez AJ, Kenyon NS, et al. (2003) Human, nonhuman primate, and rat pancreatic islets express erythropoietin receptors. Transplant 75: 1356-1360.

25. Fenjves ES, Ochoa MS, Gay-Rabinstein C, Molano RD, Pileggi A, et al. (2004) Adenoviral gene transfer of erythropoietin confers cytoprotection to isolated pancreatic islets. Transplant 77: 13-18.

26. Dahan A, Dunne A, Swartjes M, Proto PL, Heij L, et al. (2013) ARA 290 improves symptoms in patients with sarcoidosis-associated small nerve fiber loss and increases corneal nerve fiber density. Mol Med 19: 334-345.

27. Brines M, Dunne AN, van Velzen M, Proto PL, Ostenson CG, et al. (2014) ARA 290, a nonerythropoietic peptide engineered from erythropoietin, improves metabolic control and neuropathic symptoms in patients with type 2 diabetes. Mol Med 20: 658-666.

28. Muller C, Yassin K, Li LS, Palmblad M (2015) ARA290 improves insulin release and glucose tolerance in type 2 diabetic GK rats. Mol Med 2: 15-18.

29. Watanabe M, Lundgren T, Saito $Y$, Cerami A, Brines M, et al. (2016) A Nonhematopoietic Erythropoietin Analogue, ARA 290, Inhibits Macrophage Activation and Prevents Damage to Transplanted Islets. Transplant 100: 554562.

30. Sharma A, Sörenby A, Wernerson A, Efendic S, Kumagai-Braesch M, et al. (2006) Exendin-4 treatment improves metabolic control after rat islet transplantation to athymic mice with streptozotocin-induced diabetes. Diabetologia 49: 1247-1253.

31. Pileggi A, Ribeiro MM, Hogan AR, Molano RD, Cobianchi L, et al. (2009) Impact of pancreatic cold preservation on rat islet recovery and function. Transplantation 87: 1442-1450.

32. Mosmann T (1983) Rapid colorimetric assay for cellular growth and survival: application to proliferation and cytotoxicity assays. J Immunol Methods 65: 55-63.
33. Alexander M, Krishnan R, Buder B, Lamb M, Laugenour K, et al. (2014) Impact of hypothermic preservation on tissue yield and viability in pig pancreata. Transplant Proc 46: 1975-1977.

34. Emamaullee JA, Shapiro AM (2007) Factors influencing the loss of beta-cell mass in islet transplantation. Cell Transplant 16: 1-8.

35. Collino M, Thiemermann C, Cerami A, Brines M (2015) Flipping the molecular switch for innate protection and repair of tissues: Long-lasting effects of a nonerythropoietic small peptide engineered from erythropoietin. Pharmacol Ther 151: $32-40$.

36. Brines M, Patel NS, Villa P, Brines C, Mennini T, et al. (2008) Nonerythropoietic tissue-protective peptides derived from the tertiary structure of erythropoietin Proc Natl Acad Sci USA 105: 10925-10930.

37. Brines M, Cerami A (2008) Erythropoietin-mediated tissue protection: reducing collateral damage from the primary injury response. J Int Med 264: 405-432.

38. Patel NS, Kerr-Peterson HL, Brines M, Collino M, Rogazzo M, et al. (2012) Delayed administration of pyroglutamate helix B surface peptide (pHBSP), a novel nonerythropoietic analog of erythropoietin, attenuates acute kidney injury. Mol Med 18: 719-727.

39. Yang C, Zhao T, Lin M, Zhao Z, Hu L, et al. (2013) Helix B surface peptide administered after insult of ischemia reperfusion improved renal function, structure and apoptosis through beta common receptor/erythropoietin receptor and PI3K/Akt pathway in a murine model. Exp Biol Med 238: 111-119.

40. Carlsson PO, Palm F, Andersson A, Liss P (2001) Markedly decreased oxygen tension in transplanted rat pancreatic islets irrespective of the implantation site. Diabetes 50: 489-495.

41. Bennet W, Groth CG, Larsson R, Nilsson B, Korsgren O (2000) Isolated human islets trigger an instant blood mediated inflammatory reaction: implications for intraportal islet transplantation as a treatment for patients with type 1 diabetes. Ups J Med Sci 105: 125-133

42. Jaeger C, Wohrle M, Federlin K, Bretzel RG (1995) Pancreatic islet xenografts at two different transplantation sites (renal subcapsular versus intraportal): comparison of graft survival and morphology. Exp Clin Endocrinol Diabetes 103: $123-128$

43. Sakata N, Tan A, Chan N, Obenaus A, Mace J, et al. (2009) Efficacy comparison between intraportal and subcapsular islet transplants in a murine diabetic model. Transplant Proc 41: 346-349. 